\title{
Minimum Level Nonplanar Patterns for Trees
}

\author{
J. Joseph Fowler* and Stephen G. Kobourov* \\ Department of Computer Science, University of Arizona \\ $\{$ jfowler, kobourov\}@cs.arizona.edu
}

\begin{abstract}
Minimum level nonplanar (MLNP) patterns play the role for level planar graphs that the forbidden Kuratowksi subdivisions $K_{5}$ and $K_{3,3}$ play for planar graphs. We add two MLNP patterns for trees to the previous set of tree patterns given by Healy et al. 4. Neither of these patterns match any of the previous patterns. We show that this new set of patterns completely characterizes level planar trees.
\end{abstract}

\section{Introduction}

Level graphs model hierarchical relationships. A level drawing has all vertices in the same level with the same $y$-coordinates and has all edges strictly $y$-monotone. Level planar graphs have level drawings without edge crossings. Hierarchies are special cases in which every vertex is reachable via a $y$-monotone path from a source in the top level. Planar graphs are characterized by forbidden subdivisions of $K_{5}$ and $K_{3,3}$ by Kuratowksi's Theorem [5]. The counterpart of this characterization for level planar graphs proposed by Healy, Kuusik, and Liepert 44 are the minimum level nonplanar (MLNP) patterns. These are minimal obstructing subgraphs with a set of level assignments that force one or more crossings.

Di Battista and Nardelli [1] provided three level nonplanar patterns for hierarchies (HLNP patterns); cf. Fig. 2. Healy et al. adapted these HLNP patterns to MLNP patterns for level graphs. However, the completeness of their characterization was based on the claim that all MLNP patterns must contain a

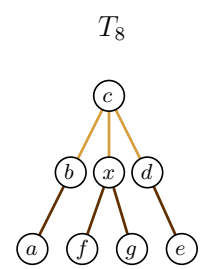

(a)

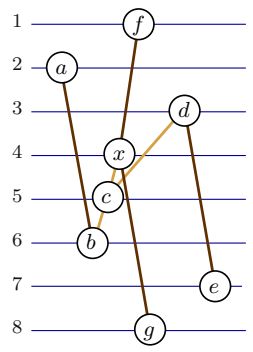

(b)

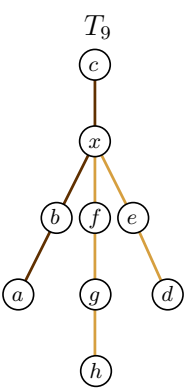

(c)

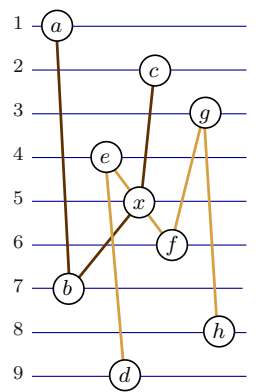

(d)

Fig. 1. Labelings preventing the forbidden ULP trees $T_{8}$ and $T_{9}$ from being level planar

\footnotetext{
* This work is supported in part by NSF grants CCF-0545743 and ACR-0222920.
} 
HLNP pattern. We provide a counterexample to this claim based on the level nonplanar assignment for the forbidden tree $T_{9}$ used by Estrella et al. [2] to characterize the set of unlabeled level planar (ULP) trees; cf. Fig. 1. Healy et al. provide two of the MLNP patterns, $P_{1}$ and $P_{2}$, for trees that are also HLNP patterns; cf. Fig. 3(a) and (b). We provide two more MLNP patterns, $P_{3}$ and $P_{4}$ for level nonplanar trees; cf. Fig. 3(c) and (d) using our counterexample.

\section{Preliminaries}

A $k$-level graph $G(V, E, \phi)$ on $n$ vertices has leveling $\phi: V \rightarrow[1 . . k]$ where every $(u, v) \in E$ either has $\phi(u)<\phi(v)$ if $G$ is directed or $\phi(u) \neq \phi(v)$ if $G$ is undirected. This leveling partitions $V$ into $V_{1} \cup V_{2} \cup \cdots \cup V_{k}$ where the level $V_{j}=\phi^{-1}(j)$ and $V_{i} \cap V_{j}=\varnothing$ if $i \neq j$. A proper level graph only has short edges in which $\phi(v)=\phi(u)+1$ for every $(u, v) \in E$. Edges spanning multiple levels are long. A hierarchy is a proper level graph in which every vertex $v \in V_{j}$ for $j>1$ has at least one incident edge $(u, v) \in E$ to a vertex $u \in V_{i}$ for some $i<j$.

A path $p$ is a non-repeating ordered sequence of vertices $\left(v_{1}, v_{2}, \ldots, v_{t}\right)$ for $t \geq 1$. Let $\min (p)=\min \{\phi(v): v \in p\}, \operatorname{MAx}(p)=\max \{\phi(v): v \in p\}$, and $\mathcal{P}(i, j)=\{p: p$ is a path where $i \leq \operatorname{MIN}(p)<\operatorname{MAX}(p) \leq j\}$ are the paths between levels $V_{i}$ and $V_{j}$. A linking path, or link, $L \in \mathcal{L}(i, j)$ is a path $x \rightsquigarrow y$ in which $i=\operatorname{MiN}(L)=\phi(x)$ and $\operatorname{MAx}(L)=\phi(y)=j$, and $\mathcal{L}(i, j) \subseteq \mathcal{P}(i, j)$ are all paths linking the extreme levels $V_{i}$ and $V_{j}$. A bridge $b$ is a path $x \rightsquigarrow y$ in $\mathcal{P}(i, j)$ connecting links $L_{1}, L_{2} \in \mathcal{L}(i, j)$ in which $b \cap L_{1}=x$ and $b \cap L_{2}=y$.

A level drawing of $G$ has all of its level- $j$ vertices in the $j^{\text {th }}$ level $V_{j}$ placed along the track $\ell_{j}=\{(x, k-j) \mid x \in \mathbb{R}\}$, and each edge $(u, v) \in E$ is drawn as a continuous strictly $y$-monotone sequence of line segments. A level drawing drawn without edge crossings shows that $G$ is level planar. A pattern is a set of level nonplanar graphs sharing structural similarities. Removing any edge from the underlying graph matching a minimum level nonplanar (MLNP) pattern gives a level planar graph. A hierarchy level nonplanar (HLNP) pattern is a level nonplanar pattern in which every matching graph is a hierarchy. The next theorem gives the set of the three distinct HLNP patterns.

Theorem 1. [Di Battista and Nardelli [1]] A hierarchy $G(V, E, \phi)$ on $k$ levels is level planar if and only if there does not exist three paths $L_{1}, L_{2}, L_{3} \in \mathcal{L}(i, j)$ linking levels $V_{i}$ and $V_{j}$ for $1 \leq i<j \leq k$ where one of the following hold:

$\left(P_{A}\right) L_{1}, L_{2}$, and $L_{3}$ are completely disjoint and pairwise connected by bridges $b_{1}, b_{2}, b_{3}$ where $b_{1} \cap L_{2}=b_{2} \cap L_{1}=b_{3} \cap L_{1}=\varnothing$; cf. Fig. Q $(a)$.

$\left(P_{B}\right) L_{1}$ and $L_{2}$ share a path $C=L_{1} \cap L_{2}$ from $p \in V_{i} \cup V_{j}$ where $L_{1} \cap L_{3}=$ $L_{2} \cap L_{3}=\varnothing$ are connected by bridges $b_{1}$ from $L_{1}$ to $L_{3}$ and $b_{2}$ from $L_{1}$ to $L_{3}$ such that $b_{1} \cap L_{2}=b_{2} \cap L_{1}=\varnothing$; cf. Fig. Q $(b)$.

$\left(P_{C}\right) L_{1}$ and $L_{2}$ share a path $C_{1}=L_{1} \cap L_{2}$ from $p \in V_{i}$ and $L_{2}$ and $L_{3}$ share $a$ path $C_{2}=L_{2} \cap L_{3}$ from $q \in V_{j}$ such that $C_{1} \cap C_{2}=\varnothing$. Bridge $b$ connects $L_{1}$ and $L_{3}$ where $b \cap L_{2}=b \cap C_{1}=b \cap C_{2}=\varnothing$; cf. Fig. [2(c). 


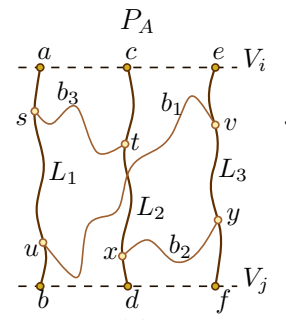

(a)

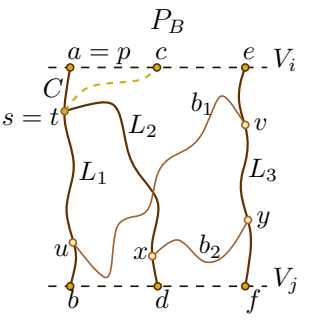

(b)

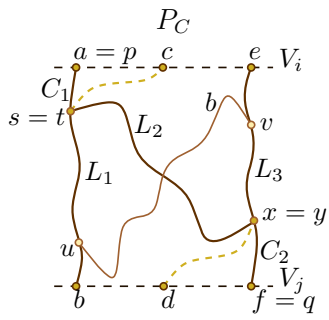

(c)

Fig. 2. The three patterns characterizing hierarchies

\section{$3 \quad$ MLNP Patterns for Trees}

Theorem 2. A level tree $T(V, E, \phi)$ on $k$ levels is minimum level nonplanar if (1) there are three disjoint paths $L_{1}, L_{2}, L_{3} \in \mathcal{L}(i, j)$ for $1 \leq i<j \leq k$ where $P_{A}$ of Theorem 1 applies and the union of the three bridges $b_{1} \cup b_{2} \cup b_{3}$ forms a subdivided $K_{1,3}$ subtree $S$ with vertex $c$ of degree 3 where either $\left(P_{1}\right) c \in V_{i}\left(\right.$ or $\left.V_{j}\right)$ and there is a leaf of $S$ in $V_{j}$ (or $\left.V_{i}\right)$ as in Fig. (3) (a) or $\left(P_{2}\right)$ one leaf of $S$ is in $V_{i}$ and another leaf of $S$ is in $V_{j}$ as in Fig. 3(b), or

(2) there are four paths $L_{1}, L_{2}, L_{3}, L_{4} \in \mathcal{L}(i, j)$ for $1 \leq i<j \leq k$ where $L_{1} \cap L_{4}=$ $\varnothing, L_{1} \cap L_{2} \in V_{j}$ (or $V_{i}$ ) and $L_{3} \cap L_{4} \in V_{i}$ (or $V_{j}$ ) where $L_{1} \cup L_{2}$ and $L_{3} \cup L_{4}$ form paths with both endpoints in $V_{i}$ and $V_{j}$ (or $V_{j}$ and $V_{i}$ ), resp., and there exist levels $V_{l}$ and $V_{m}$ for some $i<l<m<j$ in which either $L_{2}$ or $L_{3}$ consists of subpaths $C_{1} \in \mathcal{L}(i, m), C_{2} \in \mathcal{L}(l, m)$, and $C_{3} \in \mathcal{L}(l, j)$ where either $\left(P_{3}\right) L_{2} \cap L_{3}=x$ where $l \leq \phi(x) \leq m$ as in Fig. 3)(c), or

$\left(P_{4}\right) L_{2} \cap L_{3}$ is path $x \rightsquigarrow y$ where $l \leq\{\phi(x), \phi(y)\} \leq m$ and $L_{2}=c \rightsquigarrow x \rightsquigarrow$

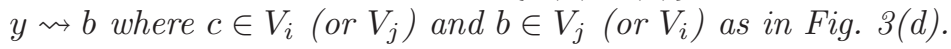

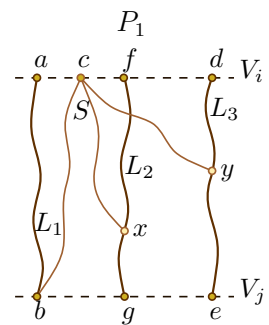

(a)

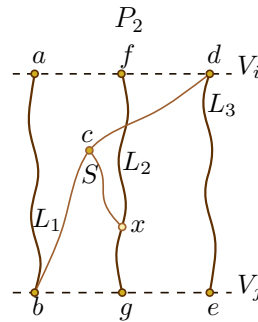

(b)

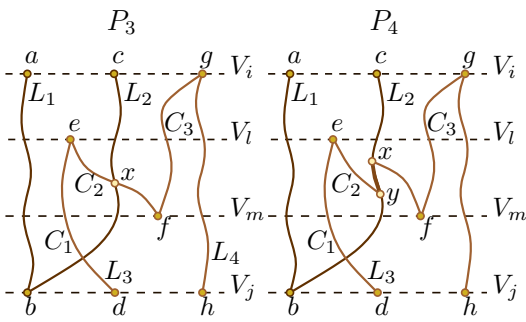

(c)

(d)

Fig. 3. Four MLNP patterns for trees

Proof. $P_{1}$ and $P_{2}$ are MLNP given they match T1 and T2 of Healy et al. The argument in 2] used by Estrella et al. to show $T_{9}$ is level nonplanar generalizes for $P_{3}$ and $P_{4}$. To see that $P_{3}$ is minimal ( $P_{4}$ is similar), we try the seven distinct ways of removing an edge; cf. Fig. 4 . In each case crossings can be avoided.

The proof of Theorem 15 of Healy et al. 4 argues that every MLNP pattern must match some HLNP pattern. We show why this argument fails for $P_{3}$. 


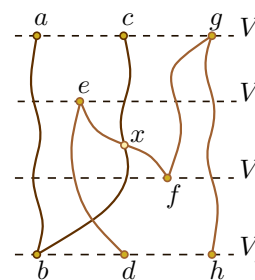

(a)

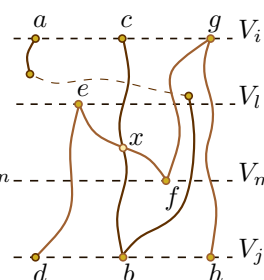

(b)

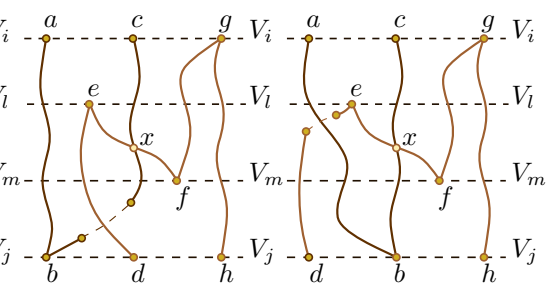

(c) (d)

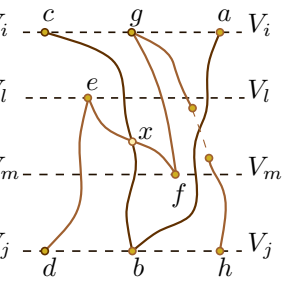

(h)

(e)

(f)

(g)

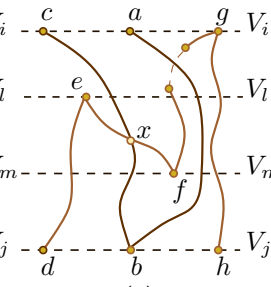

Fig. 4. The seven cases of deleting an edge from pattern $P_{3}$ in (a)

Lemma 3. $P_{3}$ augmented to form a hierarchy has a subtree matching $P_{2}$.

Proof. Fig. 5 shows the highlighted subtrees that match $P_{2}$ when $P_{3}$ is augmented to form a hierarchy. However, $P_{2}$ does not match $P_{3}$ by Theorem 2 .

The next lemma gives the minimal conditions for a MLNP tree pattern.

Lemma 4. A level nonplanar tree $T(V, E, \phi)$ on $k$ levels contains three disjoint paths $L_{1}, L_{2}, L_{3} \in \mathcal{L}(i, j)$ linking levels $V_{i}$ and $V_{j}$ for $1 \leq i<j \leq k$ with bridges $b_{1}$ from $L_{1}$ to $L_{2}$ and $b_{2}$ from $L_{2}$ to $L_{3}$ with $x=b_{1} \cap L_{2}$ and $y=b_{2} \cap L_{2}$ so that either $\left(P_{\alpha}\right) x=y,\left(P_{\beta}\right) L_{2}=c \rightsquigarrow y \rightsquigarrow x \rightsquigarrow d$, or $\left(P_{\gamma}\right) L_{2}=c \rightsquigarrow x \rightsquigarrow y \rightsquigarrow d$ hold where $c \in V_{i}$ and $d \in V_{j}$ as in Fig. G(a), (b), (c).

Proof. Assume that $P$ is an MLNP pattern between levels $V_{i}$ and $V_{j}$ in which $|i-j|$ is minimum and there are at most two disjoint paths $L_{1}, L_{2} \in \mathcal{L}(i, j)$.

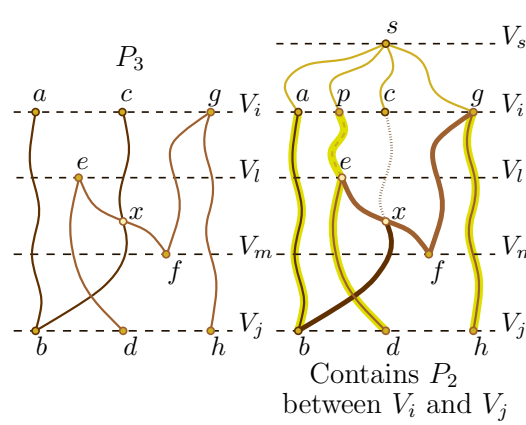

(a) (b)
Contains $P_{2}$ between $V_{l}$ and $V_{j}$

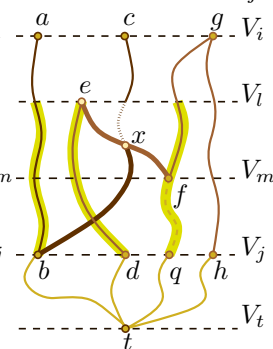

(c)

Fig. 5. Augmenting $P_{3}$ in (a) from above (b) and below (c) to form hierarchies 


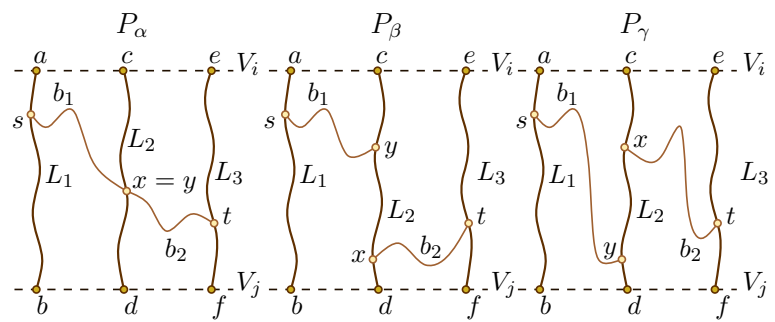

(a) (b)

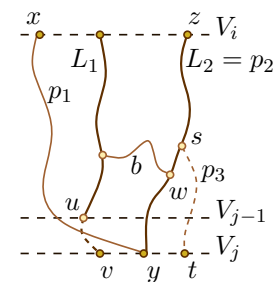

(d)

Fig. 6. Minimal patterns for Lemma 4

There could be at most one bridge $b$ joining $L_{1}$ and $L_{2}$ without forming a cycle. Let $w$ be the endpoint of $b$ in $L_{2}$. Let $P^{\prime}$ be $P-(u, v)$ where $(u, v)$ is the short edge connecting $L_{1}$ to $V_{j}$ in which $v \in V_{j}$. In order for $P$ to be MLNP, there must exist two linking paths $p_{1}, p_{2} \in \mathcal{L}(i, j)$ in $P^{\prime}$ with endpoints $x, z \in V_{i}$ and common endpoint $y \in V_{j}$ such that for any level planar embedding of $P^{\prime}, u$ is contained in the region bounded by $p_{1}, p_{2}$ and the track $\ell_{i}$; cf. Fig. 6)(d). Assume w.l.o.g. that $L_{2}$ is $p_{2}$. In order for $p_{1}$ not to be embeddable on the other side of $p_{2}$ (allowing edge $(u, v)$ to be drawn in $P$ without crossing), there must be a path $p_{3}$ from $s$ in $L_{2}$ to $t \in V_{j}$ in which $s$ lies between $z$ and $w$ blocking this direction. Then there are at least three disjoint paths in $P$ in $\mathcal{L}(i, j): p_{1}, L_{1}$ and the path $z \rightsquigarrow s \rightsquigarrow t$, contradicting our assumption of there only being two.

Let $L_{1}, L_{2}, L_{3} \in \mathcal{L}(i, j)$ be three disjoint paths. At least one of the three paths, say it is $L_{2}$, must be joined by bridges $b_{1}$ and $b_{2}$ to the other two paths $L_{1}$ or $L_{3}$, respectively, or $P$ would be disconnected contradicting the minimality of $P$. If $b_{1} \cap b_{2}$ form a nonempty path, then $b_{1} \cup b_{2}$ would form a subtree homeomorphic to $K_{1,3}$, yielding pattern $P_{1}$ or $P_{2}$ of Theorem 2. Thus, $b_{1}$ and $b_{2}$ can share at most one vertex as in $P_{\alpha}$ of Fig. 6(a). Otherwise there must have been endpoints $x=b_{1} \cup L_{2}$ and $y=b_{2} \cup L_{2}$ along the path $c \rightsquigarrow d$ forming $L_{2}$ where either $y$ proceeds $x$ as in $P_{\beta}$ of Fig. 6(b) or $x$ proceeds $y$ as in $P_{\gamma}$ of Fig. 6(c).

We next show that $P_{4}$ is easily derived from $P_{3}$.

Lemma 5. $P_{4}$ is the only distinct MLNP pattern for trees that can be formed from $P_{3}$ (by splitting the degree-4 vertex) not containing a subtree matching $P_{2}$.

Proof. Fig. 7 shows the three ways the degree- 4 vertex of $P_{3}$ can be split into two degree-3 vertices. Two contain subtrees that match $P_{2}$.

Finally we complete our characterization for level nonplanar trees.

Theorem 6. A level tree $T$ is level nonplanar if and only if $T$ has a subtree matching one of the minimum level nonplanar patterns $P_{1}, P_{2}, P_{3}$, or $P_{4}$.

Proof Sketch: We sketch proof for the simplest case here; the full proof can be found in 3]. Once a MLNP pattern $P$ is augmented to form a hierarchy, one of the HLNP patterns must apply. Since this augmentation does not introduce a 


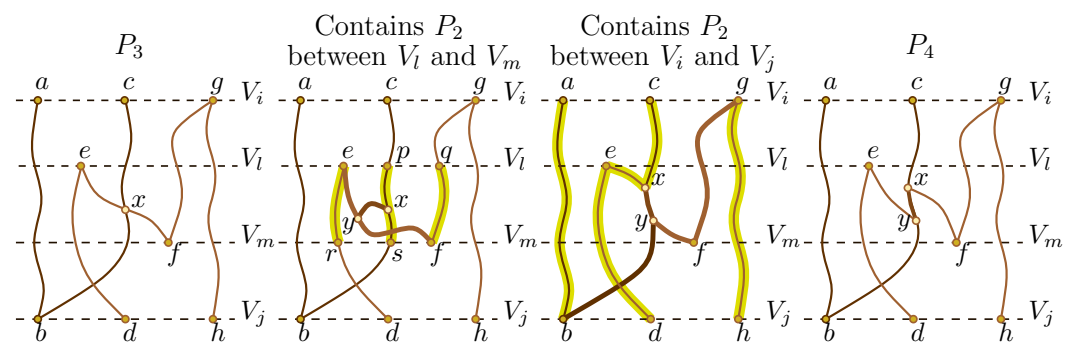

(a)

(b)

(c)

(d)

Fig. 7. The three ways of splitting the degree- 4 vertex of $P_{3}$ into two vertices of degree 3

cycle between levels $V_{i}$ and $V_{j}$, either pattern $P_{1}$ or $P_{2}$ must match a subtree of the augmented pattern by Lemma 5 of 4 .

Assume there is a MLNP tree pattern $P$ containing $P_{\alpha}$ of Lemma 4 that does not match $P_{1}$ or $P_{2}$. We consider the simplest case of how the bridges of $P_{\alpha}$ in $P$ could spans levels between $V_{i}$ and $V_{j}$. We augment $P$ to form a hierarchy to illustrate how either $P$ must match $P_{1}$ or $P_{2}$ or contain a cycle.

Suppose that a bridge of $P_{\alpha}$ in $P$ is not strictly $y$-monotone. Then $P$ could either have a bend at $e$ in level $V_{l}$ in one bridge or a bend at $f$ in level $V_{m}$ in the other as in Fig. 8(a) for some $i<l<m<j$. Each bend would require augmentation to a path from the source when forming a hierarchy from above or below as was the case with $P_{3}$ in Fig. 5.

We augment $P$ with a path $p \rightsquigarrow e$ from $V_{i}$ to $V_{l}$ to form $P^{\prime}$, a hierarchy, that must match $P_{1}$ or $P_{2}$. We observe that between levels $V_{i}$ and $V_{m}$, we have four linking paths. A third bridge $u \rightsquigarrow v$ must be present in $P^{\prime}$ that is part of a subtree $S$ homeomorphic to $K_{1,3}$. Fig. 8(b) gives one such example. While $P^{\prime}$ matches $P_{2}$ between levels $V_{i}$ and $V_{m}$, we see that between levels $V_{i}$ and $V_{j}, P$ must have had the cycle $u \rightsquigarrow v \rightsquigarrow e \rightsquigarrow b \rightsquigarrow u$, contradicting $P$ being a tree pattern. By inspection, any other placement of $u \rightsquigarrow v$ to connect three of the four linking paths to form $P_{1}$ or $P_{2}$ similarly implies a cycle in $P$.

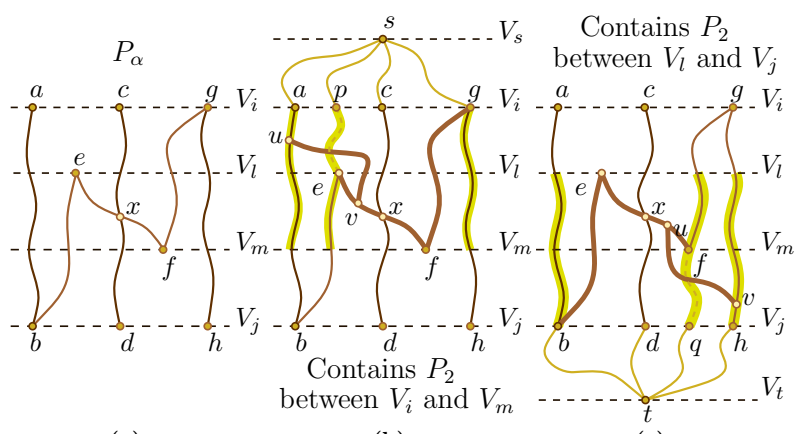

(a)

(b)

(c)

Fig. 8. Examples of pattern $P_{\alpha}$ in (a) being augmented to form a hierarchy in (b) and (c) 
Hence, $P$ cannot contain any more edges than those of $P_{\alpha}$ without matching $P_{1}$ or $P_{2}$. We observe that $P_{\alpha}$ consists of two paths sharing a common vertex $x$. Given the minimality of $P$ in minimizing $|i-j|$, one path has both endpoints in $V_{i}$ with one vertex in $V_{j}$ that can be split into linking paths $L_{1}, L_{2} \in \mathcal{L}(i, j)$. Similarly, the other has both endpoints in $V_{j}$ with one vertex in $V_{i}$ that can also be split into the linking paths $L_{3}, L_{4} \in \mathcal{L}(i, j)$. In $P_{3}$ of Fig. 8(a), $L_{1}$ is $a \rightsquigarrow b$, $L_{2}$ is $b \rightsquigarrow e \rightsquigarrow x \rightsquigarrow c, L_{3}$ is $d \rightsquigarrow x \rightsquigarrow f \rightsquigarrow g$, and $L_{4}$ is $g \rightsquigarrow h$.

For $P$ to be level nonplanar, a crossing must be forced between these two paths. This is done by having $L_{2}$ or $L_{3}$ meet the condition of $P_{3}$ of three subpaths $C_{1} \in \mathcal{L}(i, m)$ linking $V_{i}$ to $V_{m}, C_{2} \in \mathcal{L}(l, m)$ linking $V_{l}$ to $V_{m}$, and $C_{3} \in \mathcal{L}(l, j)$ linking $V_{l}$ to $V_{j}$. This is not the case for $P_{\alpha}$ in Fig. 8 (a) since the $x \rightsquigarrow c$ portion of $L_{2}$ does not reach level $V_{m}$, and the $x \rightsquigarrow d$ portion of $L_{3}$ does not reach level $V_{l}$. So for $P$ not to match $P_{3}$, at least one subpath of both $L_{2}$ and $L_{3}$ from $x$ to $V_{i}$ or $V_{j}$ must strictly monotonic as was the case in Fig. 8(a). However, in this case $P$ can be drawn without crossings. This leaves $P_{3}$ as the only possibility of a MLNP pattern matching $P_{\alpha}$ that does not match $P_{1}$ or $P_{2}$.

\section{References}

1. Di Battista, G., Nardelli, E.: Hierarchies and planarity theory. IEEE Trans. Systems Man Cybernet 18(6), 1035-1046 (1988)

2. Estrella-Balderrama, A., Fowler, J.J., Kobourov, S.G.: Characterization of unlabeled level planar trees. In: Kaufmann, M., Wagner, D. (eds.) GD 2006. LNCS, vol. 4372, pp. 367-369. Springer, Heidelberg (2007)

3. Fowler, J.J., Kobourov, S.G.: Minimum level nonplanar patterns for trees. Technical Report TR07-04, University of Arizona (2007), ftp://ftp.cs.arizona.edu/reports/2007/TR07-04.pdf

4. Healy, P., Kuusik, A., Leipert, S.: A characterization of level planar graphs. Discrete Math. 280(1-3), 51-63 (2004)

5. Kuratowski, C.: Sur les problèmes des courbes gauches en Topologie. Fundamenta Mathematicae 15, 271-283 (1930) 